\title{
THE STABILITY OF DE RHAM CURRENTS
}

\author{
MIRCEA PUTA
}

ABstract. The purpose of this short note is to give a theorem concerning the action of the diffeomorphism group of a smooth manifold on the space of de Rham currents.

Let $M$ be a paracompact, orientable $n$-dimensional, $C^{\infty}$-manifold. We shall denote by $\mathscr{D}^{\prime p}(M)$ [resp. $\mathscr{Z}^{\prime p}(M)$ ] the space of de Rham currents [resp. closed de Rham currents] on $M$, endowed with the uniform convergence topology [resp. induced topology] [3].

It is easy to see that the notions of local and global stability for differential forms [1], [2] can be extended, in natural way, for de Rham currents.

The following theorem shows that there exist no globally defined stable de Rham currents on $M$.

TheOREM. Let $T \in \mathscr{D}^{\prime p}(M)$. Then for any neighborhood $V_{T}$ of $T$ there exists a p-current $S$ in $V_{T}$ such that for any smooth diffeomorphism $f: M \rightarrow M$ one has $f(S) \neq T$.

THE SKETCH OF THE PROOF. We shall suppose that the theorem is not true. First one considers the case when $T$ is a form-like de Rham current (i.e., a current induced by a form), and then the case when $T$ is not a form-like de Rham current. Using the density of Dirac currents for the first case and the density of form-like currents for the second case, one obtains immediately a contradiction. Q.E.D.

So the notion of global stability for de Rham currents does not make sense.

REMARK. Assuming that $M$ is a compact manifold without boundary, and taking into account the Hodge-Kodaira-de Rham decomposition theorem [3], the above theorem is also true if one replaces $\mathscr{D}^{\prime p}(M)$ with $\mathscr{Z}^{\prime P}(M)$.

\section{BIBLIOGRAPHY}

1. M. Golubitsky and D. Tischler, The nonexistence of globally stable forms, Proc. Amer. Math. Soc. 58 (1976), 296-300.

2. J. Martinet, Sur les singularités des formes différentielles, Ann. Inst. Fourier (Grenoble) 20 (1970), 95-178.

3. G. de Rham, Variétés différentiables. Formes, courants, formes harmoniques, Actualités Sci. Ind., no. 1222 = Publ. Inst. Math. Univ. Nancago III, Hermann, Paris, 1955.

University of Tmisoara, Seminarul de Geometrie Topologie, 1900 Tmisoara, Romania

Received by the editors December 30, 1979.

1980 Mathematics Subject Classification. Primary 58A25.

(c) 1980 American Mathematical Society $0002-9939 / 80 / 0000-0584 / \$ 01.25$ 